\title{
Influence of a Circular Ionospheric Depression on VLF Propagation
}

\author{
James R. Wait
}

\section{Contribution From the Central Radio Propagation Laboratory, National Bureau of Standards, Boulder, Colo.}

\author{
(Received April 1, 1964)
}

\begin{abstract}
Propagation of VLF radio waves in the earth-ionosphere waveguide is considered for the situation where the ionosphere is depressed over a circular region. Using first-order scattering theory, expressions for the expected field perturbations are developed in the form of double integrals. In a number of important special cases, these are expressed in closed form. In certain other situations, the integrations are carried out by numerical methods. The results confirm that a localized ionospheric depression may modify the received field even though the ionosphere along the great circle path is undisturbed.
\end{abstract}

\section{Introduction}

In a previous paper [Wait, 1964], an approximate analysis was given for VLF propagation in the earth ionosphere waveguide of variable height. The height changes were not restricted to points along the great circle path connecting transmitter and receiver. By choosing a simple example, it was shown that ionospheric perturbations lying within the first Fresnel zone are most effective in modifying the phase of the signal. In this paper, the ionosphere is assumed to be depressed in the form of a shallow inverted bowl. Such a condition may exist as a result of a localized ionizing source such as a high altitude nuclear explosion.

The method employed in the previous paper [Wait, 1964] is followed here. It is based on the idea that the phase velocity of the waveguide modes is determined by the local value of the ionospheric height. This concept has also been used by Crombie [1964] in treating similar problems in radio propagation under disturbed conditions. The problem thus becomes a two-dimensional one, and it may be readily demonstrated that there is a close analogy with weak scattering from cylindrical obstacles. An inherent complication is the near field character of the problem. This will be evident in what follows.

\section{Formulation}

The situation is illustrated in figure 1 which is a plan view of the circular disk-shaped region. Choosing a Cartesian coordinate system, $(x, y)$, the vertical dipole transmitter is located at $T\left(-x_{1}, 0\right)$, and the receiver is located at $R\left(x_{0}, 0\right)$. Furthermore, without loss of generality, the center $Q$ of the disk region is on the $y$ axis with coordinates $\left(0, y_{0}\right)$. As far as the path geometry is concerned, the earth's surface is regarded as flat. The vertical field $e$ at $R$ may

1 This work has been supported by the Advanced Research Projects Agency, Washington, D.C., under A RPA Order No. 183-62.

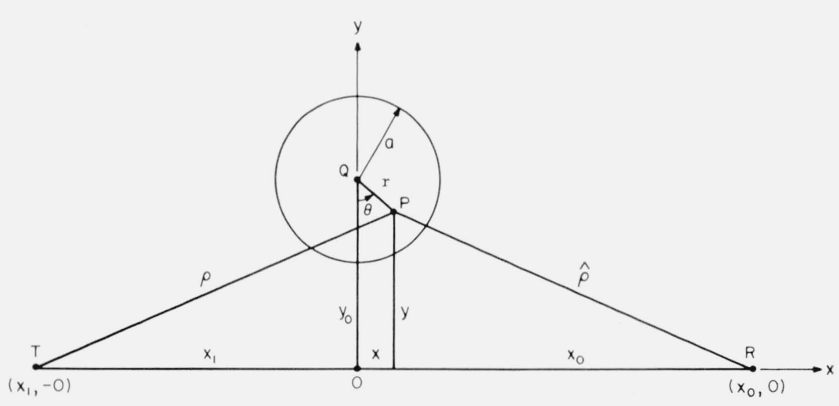

Figure 1. Plan view of the situation.

be regarded as the sum of the primary field $e^{0}$ and the secondary field $e^{s}$, which is scattered from the disk region. Following the reasoning given in the previous paper [Wait, 1964], it is found that

$$
\begin{aligned}
& e^{s}=e-e^{0} \cong-\frac{i k^{2}}{4} \iint {\left[S^{2}(x, y)\right.} \\
&\left.-\left(S^{0}\right)^{2}\right] e(x, y) H_{0}^{(2)}\left(k S^{0} \hat{\rho}\right) d x d y,
\end{aligned}
$$

where

$$
\rho=\left[\left(x_{1}+x\right)^{2}+y^{2}\right]^{1 / 2} \text { and } \hat{\rho}=\left[\left(x_{0}-x\right)^{2}+y^{2}\right]^{1 / 2} .
$$

$H_{0}^{(2)}$ is the Hankel function of the second kind of order zero, $k S(x, y)$ is the (complex) wave number for a particular waveguide mode at a point $P$ within the disk region, and $k S^{0}$ is the (complex) wave number for the same waveguide mode outside the disk region. The integration extends over the region where $S(x, y)$ differs from $S^{0}$, namely, the disk region. The complex quantities $S$ and $S^{0}$ may be computed from the theory of VLF mode propagation [Wait, 1962a].

The above general result is really an integral equation for the resultant field $e$. As a consequence of the two-dimensional formulation, it is understood that such an equation holds for each waveguide 
mode. Because of the slowly varying nature of the problem, the coupling between these equations is absent. In other words, it is assumed that mode conversion is negligible, which is justified when the cross section of the guide changes slightly in a lateral distance of one wavelength [Wait, 1962b].

\section{Simplification of Double Integral}

The first order (Born-type) approximation corresponds to the replacement of $e(x, y)$, within the integral of $(1)$, by $e^{0}(x, y)$. Furthermore, $\rho$ and $\hat{\rho}$, where they occur in the phase factors, are expanded binomially and terms beyond the second order in $y^{2}$ are neglected. In the amplitude factors, $\rho$ and $\hat{\rho}$ are replaced simply by $\left(x_{1}+x\right)$ and $\left(x_{0}-x\right)$, respectively. Thus, as indicated in the previous paper [Wait, 1964],

$$
\begin{aligned}
\frac{e^{s}}{e^{0}} \cong-\frac{i k^{2}}{4} \iint & {\left[\frac{2 i}{\pi k S_{0}\left(x_{0}-x\right)}\right]^{\frac{1}{2}} } \\
& {\left[S^{2}-\left(S^{0}\right)^{2}\right]\left[\frac{x_{0}+x_{1}}{x_{1}+x}\right]^{\frac{1}{2}} e^{-i \alpha^{2} y^{2}} d x d y, }
\end{aligned}
$$

where

$$
\alpha=k^{\frac{1}{2}} S^{0}\left[\frac{\left(x_{1}+x_{0}\right)}{2\left(x_{0}-x\right)\left(x_{1}+x\right)}\right]^{\frac{1}{2}} \text {. }
$$

The above result may be conveniently rewritten in the form

where

$$
\frac{e^{s}}{e^{0}}=-i k \int_{-a}^{+a} \Omega(x) d x,
$$

$$
\Omega(x)=\alpha\left(\frac{i}{\pi}\right)^{\frac{1}{2}} \int_{y_{1}(x)}^{y_{2}(x)}\left[S(x, y)-S^{0}\right] e^{-i \alpha^{2} y^{2}} d y
$$

and it is understood that $y_{1}$ and $y_{2}$ are the upper and lower limits of the $y$ integration. In writing the latter result, use is made of the fact that $S$ and $S^{0}$ are near unity for the important modes.

In order to obtain some sort of convenient normalization, it is desirable to first imagine that the disk region straddles the path. Also, it is assumed that $y_{2}(x) \rightarrow \infty$ and $y_{1}(x) \rightarrow-\infty$, which readily leads to

and thus,

$$
\Omega(x)=\Omega_{\infty}(x)=S(x, 0)-S^{0},
$$

$$
\left.\frac{e^{s}}{e^{0}}=\frac{e^{s}}{e^{0}}\right]_{\infty} \cong-i k \int_{-a}^{a}\left[S(x, 0)-S^{0}\right] d x .
$$

It is immediately evident that the preceding simple formula corresponds to the anomaly or secondary field of a strip of width $2 a$ whose propagation wave number is $k S(x, 0)$ for $-a<x<a$. 邆 It proves to be convenient to write

$$
\left.\frac{e^{s}}{e^{0}}\right]_{\infty}=-i 2 k a\left[S(0,0)-S^{0}\right] P_{\infty},
$$

where

$$
P_{\infty}=\frac{1}{2 a} \int_{-a}^{a}\left[\frac{S(x, 0)-S^{0}}{S(0,0)-S^{0}}\right] d x
$$

is determined by the shape of the ionospheric depression. Normally, $P_{\infty}$ is somewhat less than unity since the maximum depression is in the middle of the disk region.

In the general case, one writes

$$
\frac{e^{s}}{e^{0}}=-i 2 k a\left[S(0,0)-S^{0}\right] P,
$$

where

$$
P=\frac{1}{2 a} \int_{-a}^{a}\left[\int_{y_{1}(x)}^{y_{2}(x)} f(x, y) e^{-i \alpha^{2} y^{2}} d y\right]\left(\frac{i}{\pi}\right)^{\frac{1}{2}} \alpha d x,
$$

with

$$
f(x, y)=\frac{S(x, y)-S^{0}}{S(0,0)-S^{0}}
$$

It is clear that, in the limiting case when $y_{1} \rightarrow-\infty$ and $y_{2} \rightarrow+\infty$, the quantity $P$ approaches $P_{\infty}$.

When the disk region is assumed to possess circular symmetry, it is desirable to express the double integral in terms of cylindrical coordinates $(r, \theta)$ as indicated in figure 1 . Thus, the quantity $P$ is written in the form

$$
P=\left(\frac{i}{\pi}\right)^{\frac{1}{2}} \frac{1}{2 a} \int_{0}^{a} \int_{0}^{2 \pi} f(r) e^{-i \alpha^{2} y^{2}} \alpha r d \theta d r,
$$

where

$$
\begin{gathered}
y=y_{0}-r \cos \theta, \\
\alpha \cong\left[\frac{k\left(x_{1}+x_{0}\right)}{2\left(x_{0}-r \sin \theta\right)\left(x_{1}+r \sin \theta\right)}\right]^{\frac{1}{2}},
\end{gathered}
$$

and

$$
f(r)=\frac{S(r)-S^{0}}{S(0)-S^{0}}
$$

At first glance, this appears to be more complicated than the Cartesian form of the integral. Considerable simplification is achieved, however, when it is assumed that both $x_{0}$ and $x_{1}>>a$. Thus, within this restriction, $\alpha$ is a constant given by

$$
\alpha=\left[\frac{k\left(x_{1}+x_{0}\right)}{2 x_{0} x_{1}}\right]^{\frac{1}{2}} \text {. }
$$

Then, by changing the radial variable to $B=\alpha r$, and defining $A=\alpha y_{0}$, it is found that

$$
P=\left(\frac{i}{\pi}\right)^{\frac{1}{2}} \frac{1}{2 \alpha a} \int_{0}^{\alpha a} f\left(\frac{B}{\alpha}\right) F(A, B) B d B,
$$

where

$$
F(A, B)=\int_{0}^{2 \pi} e^{-i(A+B \cos \theta)^{2}} d \theta .
$$

The major task remaining is the determination of $P$ for a specific form of the radial function $f$ $(B / \alpha)$. In general, resort must be made to numeri- 
cal methods. However, when $f(B / \alpha)$ is a constant, some special limiting cases may be expressed in closed form. The derivation for these special formulas are given in appendixes $\mathrm{A}$ and $\mathrm{B}$.

\section{Some Concrete Results for Specific Cases}

As mentioned above, the simplest case is when $f(r)$ is a constant corresponding to an ionospheric depression of unvarying height as indicated in figure 2. For this model, it is assumed that $f(r)=1$ when $r<a$ and $f(r)=0$ when $r>a$. The integrals for the functions $F(A, B)$ and $P$ were evaluated numerically for this case, by the method of Gaussian quadrature [Kopal, 1961]. As is well known, in quadrature methods, a definite integral is approximated by a weighted sum of particular values of the ordinate with abscissas distributed in an optimum manner. In actual fact, the abscissas are roots of the Legendre polynomials and the weights (of the ordinates) are functions of these roots. The resulting error may, in general, be made arbitrarily small by increasing the number of intervals which here is denoted $n$.

Because of the highly oscillatory nature of the integrals, in the equations for both $F$ and $P$, extreme care was needed to insure that the integrals converged to their proper values. ${ }^{2}$ Thus, $P$ was evaluated, for a representative set of $A$ 's, by using an arbitrary value of $n$. The value of $n$ was then increased until the resultant values of $P$ became insensitive to further changes of $n$. The value of $n=48$ was satisfactory to evaluate $P$ for $\alpha a=0.1,0.2,0.5$, and 1 for all values of $A$. It was also sufficient for $\alpha a=2$ when $A<10$, for $\alpha a=3$ when $A<5$, and for $\alpha a=5$ when $A<1$. In other cases, it was necessary to take $n=96$ except $\alpha a=5$ when $A>10$. In this latter case, 192 abscissas were needed for $F(A, B)$ and 96 for $P$.

Some important checks were carried out for special cases using the formulas derived in the appendixes. When $A=0$, for all values of $\alpha a$, the closed form given by (B-9) was found to give answers within five digits of the quadrature answers. For other values of $A$ and when $\alpha a=0.1,0.2$, and 0.5 , the approximate form for $(\alpha a)^{2}<<1$, given by (B-14), was used. Here, the agreement was well within graphical accuracy for $\alpha a=0.1$ for the whole range of $A$ and for $\alpha a=0.2$ in the range where $A<9$.

The value for $P$ given by $(B-10)$, for $A>>\alpha a$, leads to an expression for $P$ in terms of Lommel functions as indicated by (B-12). Unfortunately, the voluminous tables by Dekanosidze [1960] did not cover the range of arguments which are of interest in the present investigation. Instead, $P$ was checked directly against (B-10). Agreement, to within graphical accuracy, with the quadrature method was obtained for $\alpha a=1$ when $A>8$, for $\alpha a=2$ when $\mathrm{A}>9$, and for $\alpha a=3$ and 5 when $A>10$.

F ${ }^{2}$ The numerical techniques to handle double integrals ${ }^{\top}$ of this type are described by Mrs. Lillje C. Walters in a-Technical:Note (to be issued).
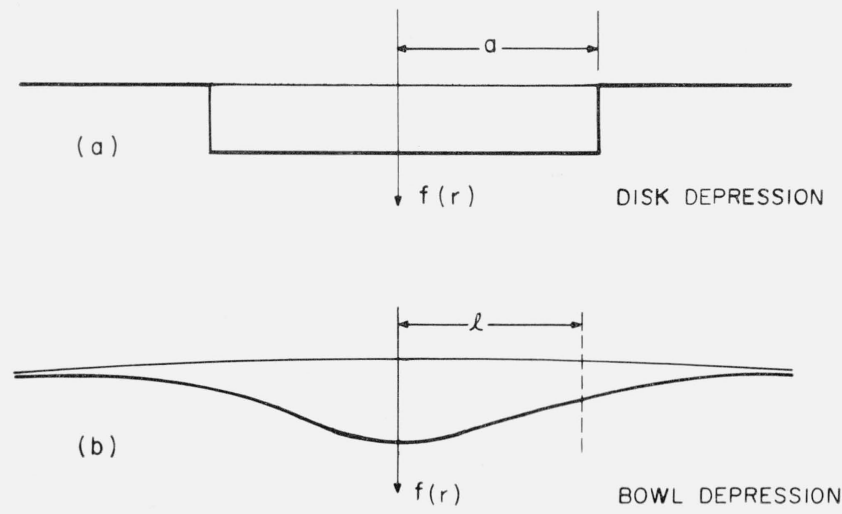

Figure 2. The form of the circular depression used in this paper.

The quadrature method of evaluating the double integrals was also employed for situations where $f(r)$ was a continuous function. For example, if $f(r)=\exp \left(-r^{2} / l^{2}\right)$ where $l$ is a constant, the method described above may be used with only minor modifications.

\section{Presentation and Discussion of Numerical Results}

As indicated by (10), the complex quantity $P$ determines the relative influence of the circularly shaped depression. To simplify the presentation here, without restricting the generality of the results, it is assumed that the quantity $\left[S(0,0)-S^{\circ}\right]$ is real. In other words, the modal attenuation over the circular region is assumed to be negligibly small. It is then clear that the real part of $P$ is related to the change of phase of the signal. Thus, following the convention in earlier work [Wait, 1964], the real part of $P$ is described as the "Normalized Phase Anomaly" or simply NPA. The imaginary part of $P$ is then related to the change of amplitude of the received field. In a similar fashion, the imaginary part of $P$ is described as the "Normalized Amplitude Anomaly" or NAA. Positive values of NAA correspond to a reduction of the total field amplitude.

Choosing $f(r)=1$ for $r\langle a$ and $f(r)=0$ for $r>a$, the depression of the ionosphere has the form shown in figure 2a. For purposes of discussion, this is called a disk-type depression. Using the methods described above, the normalized phase anomaly NPA is plotted in figure $3 \mathrm{a}$ as a function of the transverse distance parameter $A$ for various values of the factor $\alpha a$ which, itself, is proportional to the disk radius.

To facilitate the comprehension of the curves in figure 3a, two additional horizontal scales are shown. In scale I, $x_{1}=x_{0}=2000 \mathrm{~km}$ which corresponds to a separation of $4000 \mathrm{~km}$ between transmitter $T$ and receiver $R$ with the circular disk depression located midway. Thus, the scale $y_{0}$ in hundreds of $\mathrm{km}$ is the 


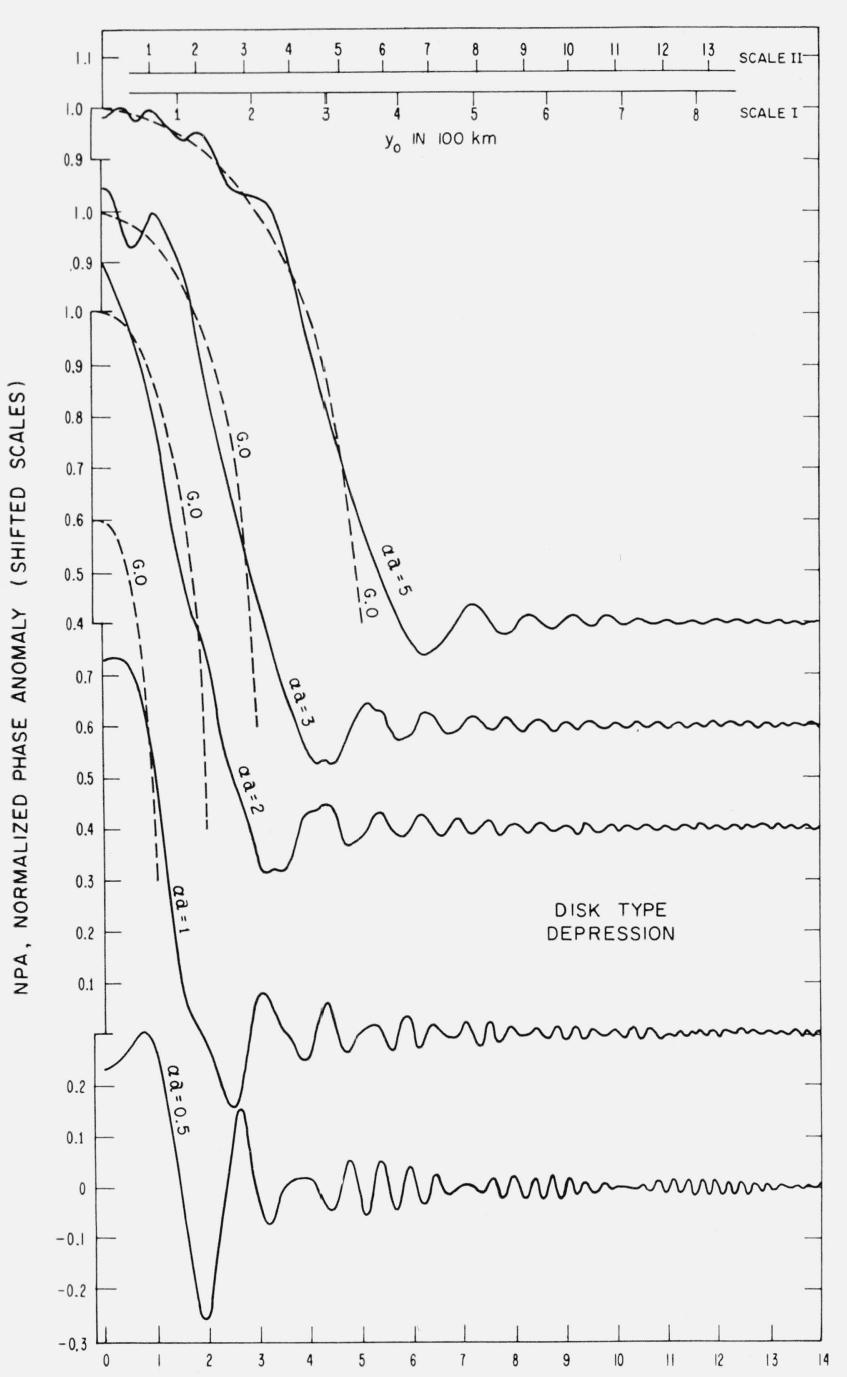

A, TRANSVERSE DISTANCE PARAMETER

Figure 3a. Normalized phase anomaly for disk depression as a function of the parameter $\mathrm{A}$ or $\alpha \mathrm{y}_{0}$.

distance from the center of the disk depression to the great circle path connecting $T$ and $R$ for a frequency of $20 \mathrm{kc} / \mathrm{s}$ (i.e., $\lambda=15 \mathrm{~km}$ ). In the inset scales in figure $3 \mathrm{a}$, the corresponding values of a are given for these same conditions. In the case of scale II, $x_{0}=$ $x_{1}=5000 \mathrm{~km}$, and the frequency remains at $20 \mathrm{kc} / \mathrm{s}$.

For the larger values of $\alpha \mathrm{a}$, it may be observed in figure 3a that NPA is approximately unity for small values of $A$. Under these circumstances, the full effect of the depression is being felt. As $A$ increases, the value of NPA diminishes and becomes very small when $A$ becomes somewhat greater than $\alpha a$.

The general behavior of the NPA curves for large values of $\alpha a$ are in general accord with simple arguments based on geometrical optics. If the depression is merely imagined to change the electrical length of the direct ray connecting the two ends of the path, an exercise in geometrical optics leads to the simple formula

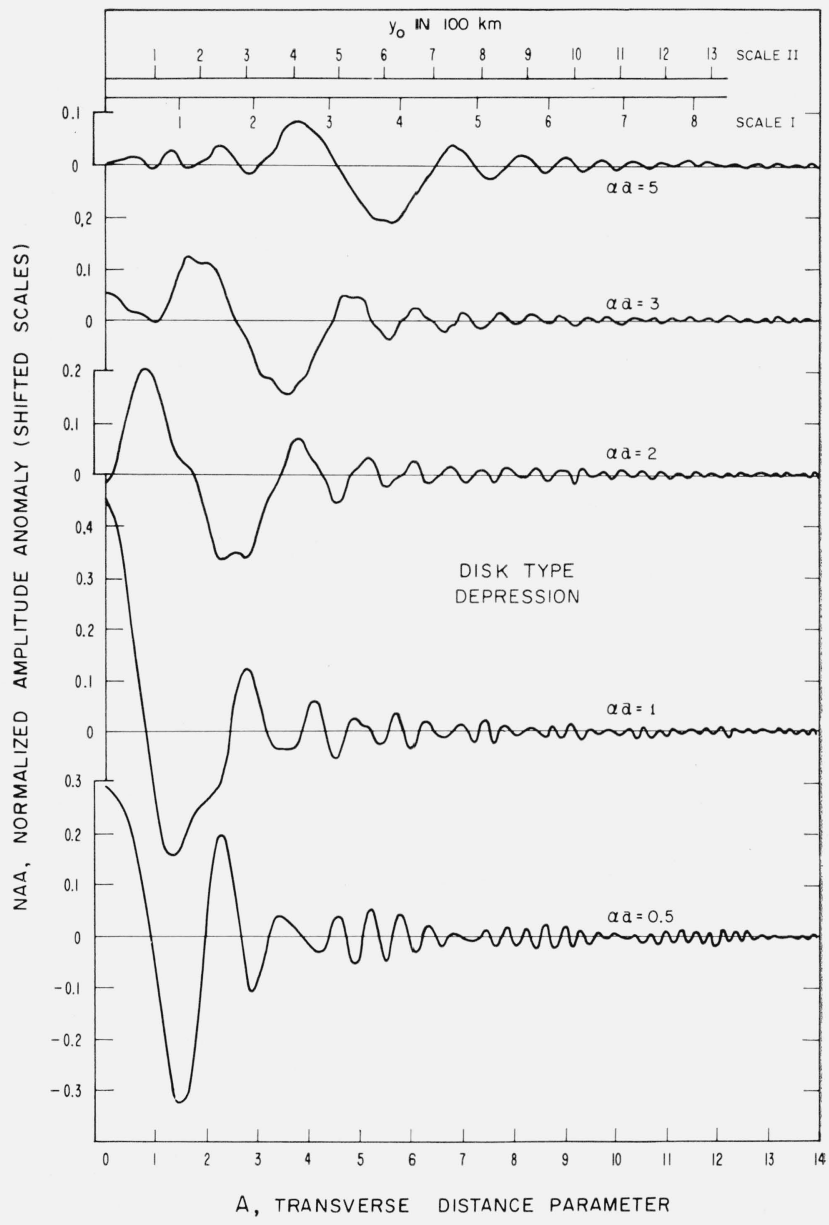

Figure 3b. Normalized amplitude anomaly for disk depression as a function of the parameter $\mathrm{A}$ or $\alpha \mathrm{y}_{0}$.

$$
\begin{aligned}
\mathrm{NPA} & =\left[1-(A / \alpha a)^{2}\right]^{\frac{1}{2}} \text { for } A<\alpha a \\
& =0 \text { for } A>\alpha a .
\end{aligned}
$$

The dashed curves corresponding to this formula are shown in figure 3a where they are labeled "G.O." to stand for "geometrical optics." As may be seen from geometry, NPA in this zero-order approximation is proportional to the chord length at a distance $y_{0}$ from the center of the circular region. It is quite amazing how well the geometrical optics predicts the shape of the central portion of the NPA curves for the larger values of $\alpha a$. Of course, geometrical optics does not give any meaningful information for values of NPA when $A$ is either comparable with $\alpha a$ or when $A$ is greater than $\alpha a$. Also, for smaller values of $\alpha a$, even though $a$ itself is several wavelengths, geometrical-optical considerations fail to give any meaningful results. In these latter situations, diffraction is playing a dominant role. 
The normalized amplitude anomaly, NAA for the disk-type depression (i.e., $f(r)=1$ ) is shown in figure $3 \mathrm{~b}$. The scales are essentially the same as those in figure $3 \mathrm{a}$. It is interesting to note that for smaller values of $\alpha a$ the value of NAA, directly behind the depression (i.e., $A=0$ ), is positive, corresponding to a diminishing of the amplitude. In this sense, the depression is acting as a diverging lense. For large values of $\alpha a$, this effect is not so clear cut.

It is interesting to note that in all cases the NAA curves have a mean value of zero with respect to the range of $A$. This factor is consistent with conservation of energy if it be remembered that the modal attenuation constant has been assumed to be negligible. Thus, the undulation of the NAA curves is a result of the redistribution of the energy which results from diffraction. The structure is complicated because of the relatively large size of the depression in terms of a wavelength.

To adopt a more realistic model of an ionospheric depression, the function $f(r)$ was taken to be a continuous function of $r$. The example chosen is the Gaussian shape defined by

$$
f(r)=\exp \left(-r^{2} / l^{2}\right),
$$

where $l$ is a constant. As a fairly good approximation, $f(r)$ shown sketched in figure $2 \mathrm{~b}$ can be regarded as the shape of the ionospheric depression. Aptly, it is described as a bowl-type depression. The assumed proportionality between the function $f(r)$ and the dependence of the ionospheric reflecting height is justified by the approximate linear dependence between height changes and phase velocity changes in VLF propagation [Wait, 1962a].

The NPA curves for the bowl-type depression are shown in figure $4 \mathrm{a}$ for various values of the parameter $\alpha l$. Here, the distance $l$, which is analogous to the radius $a$ in the disk depression, is a measure of the size of the bowl-type depression. Scales I and II, which correspond to the same conditions as previously used for the disk depression, are indicated on figure $4 \mathrm{a}$.

A striking characteristic of the NPA curves in figure $4 \mathrm{a}$ is their relative smoothness. It is only for the small values of $\alpha l$ is there any evidence of an oscillating behavior as a function of $A$. In other words, the diffraction effects for such a tapered form of depression are greatly reduced. This factor is confirmed by comparing the calculated NPA data with a simple formula based on geometrical optics. In the latter case it is assumed that NPA is proportional to the chord length weighted by the function $f(r)$. Thus, in the geometrical-optical approximation

$$
\mathrm{NPA}=\frac{1}{l} \int_{0}^{\infty} \exp \left(-r^{2} / l^{2}\right) d x
$$

where $r^{2}=x^{2}+y_{0}^{2}$. This is readily evaluated to give

$$
\mathrm{NPA}=\left(\pi^{1 / 2} / 2\right) \exp \left[-A^{2} /(\alpha l)^{2}\right],
$$

where as usual, $A=\alpha y_{0}$.

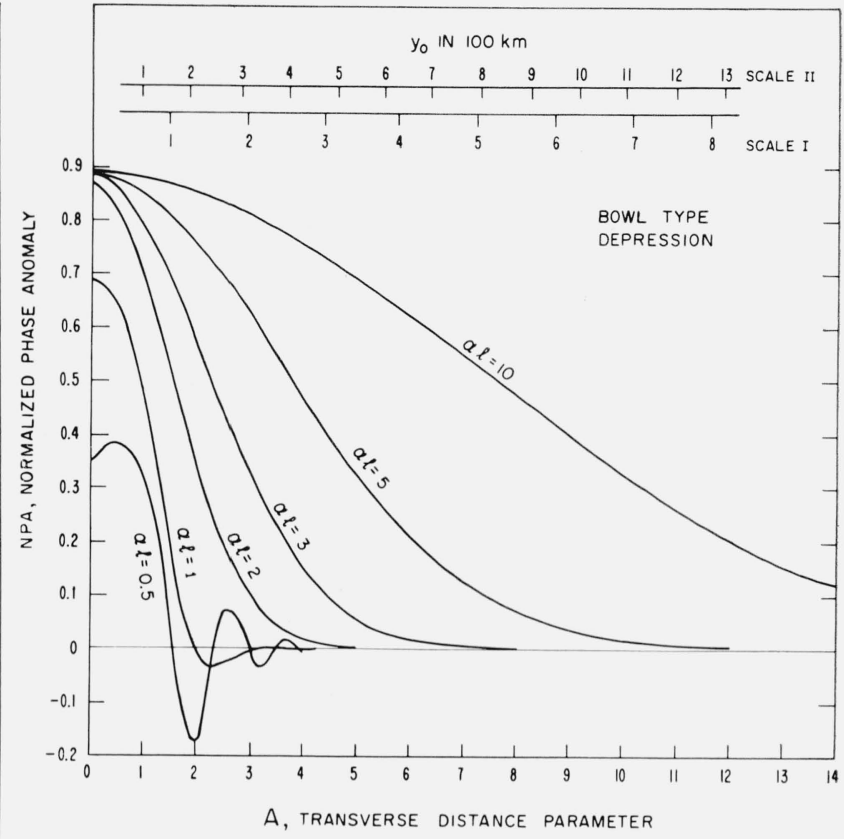

Figure 4a. Normalized phase anomaly for bowl depression as a function of the parameter $\mathrm{A}$ or $\alpha \mathrm{y}_{0}$.

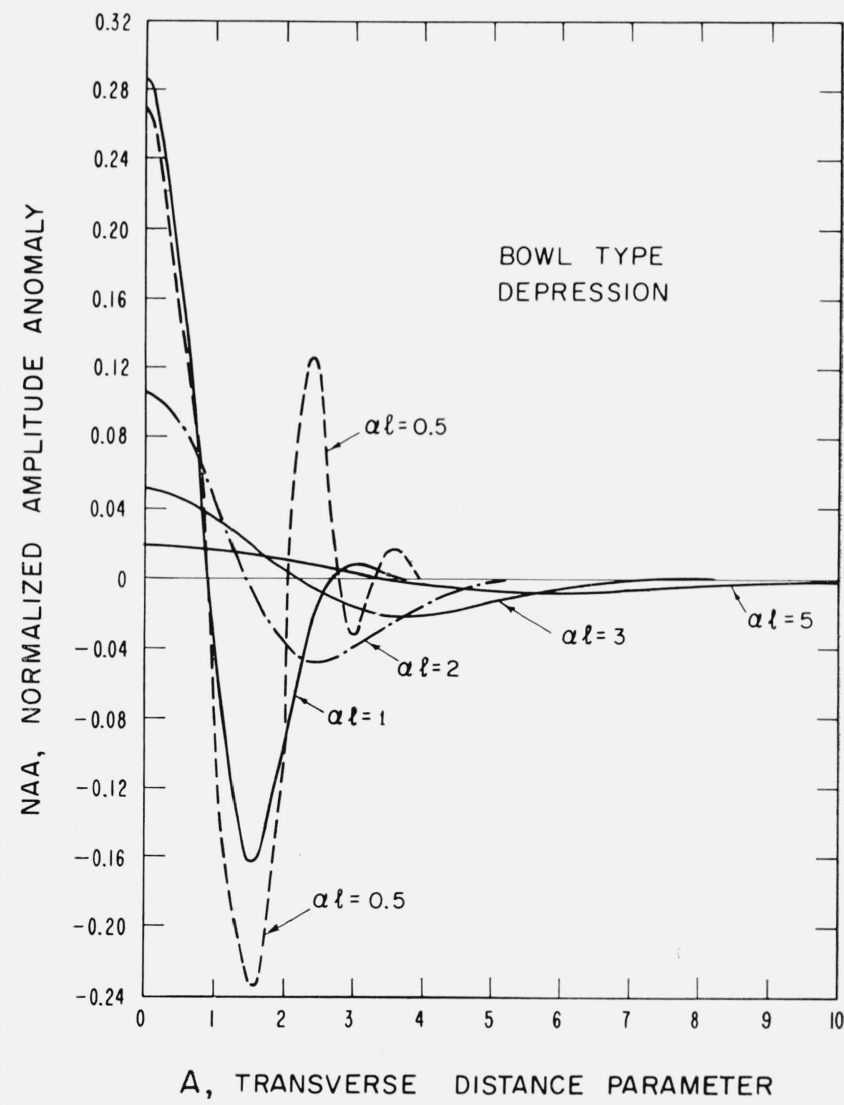

Figure 4b. Normalized amplitude anomaly for bowl depression as a function of the parameter $\mathrm{A}$ or $\alpha \mathrm{y}_{0}$. 
TABLE 1. Comparison with geometrical optics

\begin{tabular}{|c|c|c|c|}
\hline$A=-\alpha y_{\circ}$ & $\alpha l$ & NPA & $\left(\pi^{\frac{1}{2}} / 2\right) \exp \left(-A^{2} / L^{2}\right)$ \\
\hline $\begin{array}{l}0 \\
1 \\
2 \\
3\end{array}$ & $\begin{array}{l}10 \\
10 \\
10 \\
10\end{array}$ & $\begin{array}{r}0.8862 \\
.8780 \\
.8515 \\
.8000\end{array}$ & $\begin{array}{r}0.8862 \\
.8774 \\
.8515 \\
.8099\end{array}$ \\
\hline $\begin{array}{l}0 \\
1 \\
2 \\
3 \\
4 \\
5 \\
6 \\
7\end{array}$ & $\begin{array}{l}5 \\
5 \\
5 \\
5 \\
5 \\
5 \\
5 \\
5\end{array}$ & $\begin{array}{l}.8857 \\
.8510 \\
.7550 \\
.6183 \\
.4674 \\
.3260 \\
.2180 \\
.1344\end{array}$ & $\begin{array}{l}.8862 \\
.8514 \\
.7552 \\
.6183 \\
.4673 \\
.3260 \\
.2100 \\
.1248\end{array}$ \\
\hline $\begin{array}{l}0 \\
1 \\
2 \\
3 \\
4\end{array}$ & $\begin{array}{l}3 \\
3 \\
3 \\
3 \\
3 \\
3\end{array}$ & $\begin{array}{l}.8821 \\
.7909 \\
.5696 \\
.3285 \\
.1511\end{array}$ & $\begin{array}{l}.8862 \\
.7930 \\
.5682 \\
.3260 \\
.1498\end{array}$ \\
\hline $\begin{array}{l}0 \\
1 \\
2 \\
3 \\
4\end{array}$ & $\begin{array}{l}2 \\
2 \\
2 \\
2 \\
2 \\
2\end{array}$ & $\begin{array}{l}.8663 \\
.6885 \\
.3384 \\
.0964 \\
.0138\end{array}$ & $\begin{array}{l}.8862 \\
.6902 \\
.3260 \\
.0934 \\
.0162\end{array}$ \\
\hline
\end{tabular}

Within graphical accuracy, the geometrical-optical predictions for NPA are identical to the curves in figure $4 \mathrm{a}$ for $\alpha l=10$ and 5 . The departures for the curves $\alpha l=3$ and 2 are small. To indicate the correspondence, the values of NPA from the double integration are shown in table 1 along with the geometrical optical formula. The agreement for the cases of small $A$ and large $\alpha l$ is particularly good. The small but noticeable departures for large $A$ and large $\alpha l$ can probably be attributed to some convergence difficulties in the evaluation of the double integrals. On the other hand, the rather larger departures for smaller $\alpha l$ values can be attributed with some confidence to the nonvalidity of geometrical optics.

The normalized amplitude curves for the bowltype depression are shown in figure $4 \mathrm{~b}$. These are for the same conditions as the NPA curves in figure 4a. In many respects, they are analogous to the NAA curves in figure $3 \mathrm{~b}$ for the disk-type depression. The main feature of the NAA curves in figure $4 \mathrm{~b}$ is the relatively small magnitude of the ordinates.

\section{Concluding Remarks}

The results given in this paper would indicate that an artificially produced depression of the ionosphere will influence VLF propagation even though the ionosphere along the great circle path is undisturbed. The effect is most clearly noticed when the depression is sharply localized such as in the case of a circular disk-type depression with constant height. In fact, at VLF, the disturbance caused by such a depression is appreciable even when the great circle path passes within $100 \mathrm{~km}$ or more from the edge of the disk region. In such circumstances, simple ray optics would predict no disturbance.

In the case of a depression which is characterized by a tapered height distribution, the modification of the field is more in accord with geometrical-optical considerations. In this case, the diffraction effects are relatively small although, for smaller lateral dimensions of the depressed region, they are still important.

I thank Mrs. Lillie C. Walters who devised the computer program for evaluating the integrals and carried out the numerical work. The general problem was suggested by A. Glenn Jean. Some useful comments and suggestions were also received from D. D. Crombie and J. R. Johler.

\section{Appendix A. The Function $F(A, B)$}

The function $F(A, B)$ may be written in the form

$$
F(A, B)=\left(e^{-i A^{2}} / 2 \pi\right) G(A, B),
$$

where

$$
G(A, B)=\frac{1}{\pi} \int_{0}^{\pi} \exp \left[-i B^{2} \cos ^{2} \theta-2 i A B \cos \theta\right] d \theta .
$$

The form of the exponent in the latter expression suggests that we examine the generating function for Hermite polynomials [Morse and Feshbach, 1953] $H_{n}(z)$. For example,

$$
\exp \left[-t^{2}+2 z t\right]=\sum_{n=0}^{\infty} \frac{t^{n}}{n !} H_{n}(z)
$$

has the same form as the integrand of $(\mathrm{A}-2)$, if $z=-\exp (i \pi / 4) A$ and $t=\exp (i \pi / 4) B \cos \theta$. We then find

$$
G(A, B)=\sum_{n=0,1,2, \ldots}^{\infty} T_{n}(A) B^{n}
$$

where

$$
T_{n}(A)=\frac{e^{i n \pi / 4}}{(2 \pi) n !} H_{n}\left(-e^{i \pi / 4} A\right) I_{n},
$$

and

$$
\begin{aligned}
I_{n} & =\int_{0}^{2 \pi} \cos ^{n} \theta d \theta=2 \pi \frac{\Gamma\left(\frac{n+1}{2}\right)}{\Gamma\left(\frac{n}{2}+1\right)}, \quad(\mathrm{A}-6) \\
& =2 \pi\left[\frac{1 \cdot 3 \cdot 5 \cdots \cdots n-1}{2 \cdot 4 \cdot 6 \cdots \cdots n}\right] \text { for } n \text { an even integer, } \\
& =0 \text { for } n \text { odd. }
\end{aligned}
$$

Specific forms of the Hermite polynomials, $H_{n}(z)$, of low orders are: $H_{0}=1, H_{1}=2 z, H_{2}=4 z^{2}-2$, $H_{3}=8 z^{3}-12 z, \quad H_{4}=16 z^{4}-48 z^{2}+12$, and so on. Using these, the low-order values of the coefficients $T_{n}(A)$ \are igiven explicitly by $T_{0}=1, T_{1}=0, T_{2}=\frac{i H_{2}}{4}$, $T_{3}=0,{ }^{*} T_{4}=-\frac{H_{4}}{64}$, where $H_{2}=4 i A^{2}-2$, 'and $H_{4}=-16$ $-48 i A^{2}+12$.

An interesting check at this stage is to consider the special case for $A=0$. For example, 


$$
\begin{aligned}
G(0, B) & =\frac{1}{2 \pi} \int_{0}^{2 \pi} \exp \left(-i B^{2} \cos ^{2} \theta\right) d \theta \\
& =\frac{e^{-i B^{2} / 2}}{2 \pi} \int_{0}^{2 \pi} e^{-i\left(B^{2} / 2\right) \cos 2 \theta} d \theta=e^{-i B^{2} / 2} J_{0}\left(B^{2} / 2\right) .
\end{aligned}
$$

It is then readily found that

$$
\begin{aligned}
G(0, B) & =\sum_{n=0}^{\infty} T_{n}(0) B^{n} \\
& =1-\frac{i}{2} B^{2}-\frac{3}{16} B^{4}+\ldots
\end{aligned}
$$

Unfortunately, the expansion for $F(A, B)$ or $G(A, B)$, in terms of Hermite polynomials, is poorly convergent when $A$ is large. To gain some insight into the behavior of the function in this range, a saddle-point evaluation is useful. It is evident that the phase $\Omega=(A+B \cos \theta)^{2}$ is stationary when

$$
\frac{\partial \Omega}{\partial \theta}=-2(A+B \cos \theta) B \sin \theta=0 .
$$

Solutions are $\theta=0$, and $\pi$ and $\theta=\operatorname{arc} \tan (-A / B)$. When $A>>B$, it is evident that no real saddle points occur except where $\theta=0$ and $\pi$. Thus, provided $A B>>1$ and $A>>B$, it is found that, asymptotically

$$
F(A, B) \sim\left(\frac{\pi}{A B}\right)^{\frac{1}{2}}\left[e^{i \pi / 4} e^{-i(A+B)^{2}}+e^{-i \pi / 4} e^{-i(A-B)^{2}}\right] .
$$

Another special case is when $B^{2}<<1$, whence

$$
\begin{aligned}
F(A, B) & \cong \int_{0}^{2 \pi} e^{-i\left(A^{2}+2 A B \cos \theta\right)} d \theta \\
& \simeq 2 \pi e^{-i A^{2}} J_{0}(2 A B) .
\end{aligned}
$$

If, in addition, $A B>>1$, the Bessel function $J_{0}$ may be represented by the first term of its asymptotic expansion. Thus,

$$
F(A, B) \sim \frac{(\pi)^{\frac{1}{2}} e^{-i A^{2}}}{(A B)^{\frac{1}{2}}}\left[e^{i \pi / 4} e^{-i 2 A B}+e^{-i \pi / 4} e^{+2 i A B}\right],
$$

which is a special case of $(\mathrm{A}-10)$ when $B^{2}<<1$.

An approximation which combines $(\mathrm{A}-10)$ and $(\mathrm{A}-11)$ is given by

$$
F(A, B) \cong 2 \pi \exp \left[-i\left(A^{2}+B^{2}\right)\right] J_{0}(2 A B) .
$$

The latter result appears to be valid under the sole restriction that $A>>B$.

\section{Appendix B. The Radial Integration}

Using the special forms developed for $G(A, B)$ in appendix $\mathrm{A}$, the integration with respect to $B$ may be performed. For example, if (A-4 is used, it readily follows that

$$
\begin{aligned}
P & =\frac{(\pi i)^{\frac{1}{2}}}{\alpha a} e^{-i A^{2}} \int_{0}^{\alpha a} f\left(\frac{B}{\alpha}\right) G(A, B) B d B \\
& =\frac{(\pi i)^{\frac{1}{2}}}{\alpha a} e^{-i A^{2}} \sum_{n=0,1,2, \ldots}^{\infty} T_{n}(A) Q_{n},
\end{aligned}
$$

where

$$
Q_{n}=\int_{0}^{\alpha a} f\left(\frac{B}{\alpha}\right) B^{n+1} d B .
$$

If $f(B / \alpha)=1$,

$$
Q_{n}=(\alpha a)^{n+2} /(n+2),
$$

and if $f(B / \alpha)=\exp (-B / L)$,

$$
\begin{aligned}
& Q_{n}= \int_{0}^{\alpha a} \exp (-B / L) B^{n+1} d B, \\
&=-\frac{(\alpha a)^{n+1} \exp (-\alpha a / L)}{(1 / L)} \\
& \quad+\frac{(n+1)}{(1 / L)} \int_{0}^{\alpha a} B^{n} \exp (-B / L) d B \\
& \simeq \frac{(n+1) !}{(1 / L)^{n+2}} \text { if } \alpha a>>L .
\end{aligned}
$$

Because of the poor convergence, it is doubtful if the expansion in terms of the coefficients $T_{n}(A)$ is very useful. However, the special case for $A=0$ does lead to a convenient closed form for the function $P$. Under this condition,

$$
F(0, B)=2 \pi \exp \left(-i B^{2} / 2\right) \cdot J_{0}\left(B^{2} / 2\right),
$$

and thus

$P=\frac{(\pi i)^{\frac{1}{2}}}{\alpha a} \int_{0}^{\alpha a} f\left(\frac{B}{\alpha}\right) \exp \left(-i B^{2} / 2\right) J_{0}\left(B^{2} / 2\right) B d B$.

Changing the variable to $x=B^{2} / 2$, it is seen that

$$
P=\frac{(\pi i)^{\frac{1}{2}}}{\alpha a} \int_{0}^{x_{0}} f\left[\frac{(2 x)^{\frac{1}{2}}}{\alpha}\right] e^{-i x} J_{0}(x) d x .
$$

This can be expressed in closed form when $f=1$. Thus,

$P=\left(\pi x_{0} / 2\right)^{\frac{1}{2}} \exp \left[-i\left(x_{0}-\pi / 4\right)\right]\left[J_{0}\left(x_{0}\right)+i J_{1}\left(x_{0}\right)\right]$,

which may be verified by differentiation with respect to $x_{0}$. A partial check is obtained by noting that $P$ tends to unity when $x_{0}$ approaches infinity.

A fairly general approximate form for $P$ is obtained by using the representation for $F(A, B)$ given by (A-13). Thus, provided $A>>\alpha a$. 


$$
P \cong \frac{(i \pi)^{\frac{1}{2}}}{\alpha a} e^{-i A^{2}} \int_{0}^{\alpha a} J_{0}(2 A B) e^{-i B^{2}} B d B
$$

Introducing a new variable, $t=B /(\alpha a)$, and defining $w=2(\alpha a)^{2}$, it is seen that

$P \cong(i \pi)^{\frac{1}{2}}(\alpha a) e^{-i A^{2}} \int_{0}^{1} J_{0}(z t) \exp \left(-i \frac{w}{2} t^{2}\right) t d t$.

This integral may be expressed in terms of the Lommel functions [Watson, 1944], $U_{n}(w, z)$, of two variables $w$ and $z$, of order $n$. Thus,

$P=(i \pi)^{\frac{1}{2}} e^{-i A^{2}}(\alpha a) \frac{e^{-i w / 2}}{w}\left[U_{1}(w, z)+i U_{2}(w, z)\right]$.

From the basic definition of the Lommel functions, it is known that [Luke, 1962]

$$
\begin{aligned}
& U_{1}(w, z)+i U_{2}(w, z)=\left(\frac{w}{z}\right)\left(\frac{2 \pi}{w}\right)^{\frac{1}{2}} \\
& \times \exp (i w / 4) \sum_{k=0}^{\infty} i^{k}(2 k+1) J_{k+\frac{1}{2}}(w / 4) J_{2 k+1}(z) .
\end{aligned}
$$

The functions $U_{1}$ and $U_{2}$ have been tabulated [Dekanosidze, 1960] for quite a restricted range of $w$ and $z$.

A special case of the above formula for $P$ is when $(\alpha a)^{2}<<1$. Thus, only the $k=0$ term in the expansion need be retained. This leads to the relatively simple result that

$$
P \cong(i \pi)^{\frac{1}{2}} \exp \left(-i A^{2}\right) \frac{J_{1}(2 A \alpha a)}{2 A} .
$$

The above form for $P$ may be derived directly by using $(\mathrm{A}-11)$ which is valid for $B^{2}<<1$. Thus, for $f(B / \alpha)=1$, it is seen that

$$
\begin{aligned}
P \cong \frac{(i \pi)^{1 / 2}}{\alpha a} & \int_{0}^{\alpha a} J_{0}(2 A B) B d B e^{-i A^{2}} \\
& =\frac{(i \pi)^{1 / 2}}{\alpha a} \frac{1}{(2 A)^{2}} e^{-i A^{2}} \int_{0}^{2 A \alpha a} z J_{0}(z) d z,
\end{aligned}
$$

which is equivalent to (B-14) above. It is interesting to note that $(\mathrm{B}-14)$ is valid for $A=0$, provided $(\alpha a)^{2}<<1$.

\section{Appendix C. Extending the Born Approximation}

The results given in this paper are valid only within the limits imposed by the Born approximation. To indicate the nature of this restriction, it is desirable to extend the range of validity by improving the assumed form of the internal field. For example, rather than replacing $e(x, y)$ by $e^{0}(x, y)$ inside the integral of (1), it would be better to use $e^{0}(x, y) \exp [-i \Delta(x, y)]$ where $\Delta(x, y)$ is to account for the modified phase of the field when it propagates into the disturbed region. If refraction effects are ignored, it is easy to show that

$$
\begin{aligned}
\Delta(x, y) & \simeq k \sin \theta \int_{-a}^{r}\left[S(r)-S^{0}\right] d r, & & 0<\theta<\pi \\
& \cong k \sin \theta \int_{r}^{a}\left[S(r)-S^{0}\right] d r, & & \pi<\theta<2 \pi .
\end{aligned}
$$

This means that the integral $F(A, B)$, as given by (18), is to be replaced by

$$
F(A, B)=\int_{0}^{2 \pi} e^{-i \Delta(x, y)} e^{-i(A+B \cos \theta)^{2}} d \theta
$$

or

$F(A, B)=2 \int_{0}^{\pi} \cos [g(B) \sin \theta] e^{-i g_{0} \sin \theta} e^{-i(A+B \cos \theta)^{2}} d \theta$,

where

$$
g(B)=k \int_{0}^{r}\left[S(r)-S^{0}\right] d r=\frac{k}{\alpha} \int_{0}^{B}\left[S(B / \alpha)-S^{0}\right] d B,
$$

and

$$
g_{0}=k \int_{0}^{a}\left[S(r)-S^{0}\right] d r=\frac{k}{\alpha} \int_{0}^{B_{0}}\left[S(B / \alpha)-S^{0}\right] d B
$$

where $B_{0}=\alpha a$.

It is evident that this modification to the first Born approximation is insignificant when $\left|g_{0}\right|<<1$. This will be the case for small ionospheric depressions even when the extent of the depression is large in terms of the wavelength. For example, at $15 \mathrm{kc} / \mathrm{s}$, if the average value of the ionospheric depression is $10 \mathrm{~km}$, and $a=100 \mathrm{~km}$, then $g_{0}$ is only of the order of $10^{-2}$. Under some conditions, however, $g_{0}$ may be comparable with unity and corrections should be made.

\section{References}

Crombie, D. D. (May 1964), The effects of a small local change in phase velocity on the propagation of a VLF radio signal, Radio Sci. J. Res. NBS/USNC-URSI 68D, No, 6, 709-716

Dekanosidze, E. N. (1960), Tables of Lommel functions of two variables (Pergamon Press, London).

Kopal, Z. (1955), Numerical analysis (John Wiley and Sons, Inc., New York, N.Y.).

Luke, Y. L. (1962), Integrals of Bessel functions, p. 310 (McGraw-Hill Book Co., Inc., New York, N.Y.).

Morse, P. M., and H. Feshback (1953), Methods of theoretical physics, p. 786 (McGraw-Hill Book Co., Inc., New York, N.Y.).

Wait, J. R. (1962a), Electromagnetic waves in stratified media, ch. 7 (Macmillan and Co., New York, N.Y.).

Wait, J. R. (July-Aug. 1962b), An analysis of VLF mode propagation for a variable ionosphere height, J. Res. NBS 66D (Radio Prop.), No. 4, 453-461.

Wait, J. R. (Feb. 1, 1964), On phase changes in VLF propagation induced by an ionospheric depression of finite extent, J. Geophys. Res. 69, No. 3, 441-446.

Watson, G. N. (1944), Theory of Bessel functions, 2d ed., p. 537 et seq. (Cambridge Univ. Press, New York, N.Y.).

(Paper 68D8-389) 